\title{
Control of bacterial biofilm growth on surfaces by nanostructural mechanics and geometry
}

\section{Citation}

Epstein, A K, A I Hochbaum, Philseok Kim, and J Aizenberg. 2011. “Control of Bacterial Biofilm Growth on Surfaces by Nanostructural Mechanics and Geometry." Nanotechnology 22 (49)

(November 21): 494007. doi:10.1088/0957-4484/22/49/494007.

\section{Published Version}

doi:10.1088/0957-4484/22/49/494007

\section{Permanent link}

http://nrs.harvard.edu/urn-3:HUL.InstRepos:31756449

\section{Terms of Use}

This article was downloaded from Harvard University's DASH repository, and is made available under the terms and conditions applicable to Open Access Policy Articles, as set forth at http:// nrs.harvard.edu/urn-3:HUL.InstRepos:dash.current.terms-of-use\#OAP

\section{Share Your Story}

The Harvard community has made this article openly available.

Please share how this access benefits you. Submit a story.

\section{Accessibility}


Control of bacterial biofilm growth on surfaces by nanostructural mechanics and geometry

A K Epstein, A I Hochbaum, P Kim, J Aizenberg

\begin{abstract}
As the dominant mode of bacterial existence, slimy surface-associated biofilms pervade natural and anthropogenic environments. Mature biofilms can be highly resistant to liquid or vapor antimicrobial attack and therefore pose persistent pathogenic threats. Surface chemistry treatments to inhibit biofilm growth have been found to only transiently affect initial attachment. In this work, we investigate the tunable effects of physical surface properties, including high-aspect-ratio (HAR) surface nanostructure arrays recently reported to induce long-range spontaneous spatial patterning of bacteria on the surface. The functional parameters and length scale regimes that control such artificial patterning for the rod-shaped pathogenic species Pseudomonas aeruginosa are elucidated through a combinatorial approach. We further report a crossover regime of biofilm growth on a HAR nanostructured surface versus the nanostructure effective stiffness. When the "softness" of the hair-like nanoarray is increased beyond a threshold, biofilm growth is inhibited as compared to a flat control surface. This is consistent with the mechanoselective adhesion of bacteria, a recently proposed mechanism previously considered unique to eukaryotic cells. Therefore by combining nanoarray-induced bacterial patterning and modulating the effective stiffness of the nanoarray - thus mimicking an extremely compliant flat surface - bacterial mechanoselective adhesion can be exploited to control and inhibit biofilm growth.
\end{abstract}

\title{
1. Introduction
}

Bacteria often form structured, multicellular communities, called biofilms, on surfaces in natural and anthropogenic environments. [1] Biofilms contaminate a wide variety of infrastructure, such as plumbing, oil refineries, paper mills, heat exchangers, medical implants, and building HVAC systems. [2] Marine fouling, which is precipitated by the accumulation of bacterial biofilm on ship hulls followed by progressively larger marine organisms, increases the fuel expenditure of seafaring vessels by up to $40 \%$. [3] And in medical settings, biofilms are the cause of persistent infections, triggering immune response, release of harmful toxins, and even obstructing indwelling catheters. As a result, hospital-acquired (or nosocomial) infections affect about $10 \%$ of patients in the United States, accounting for nearly 100,000 deaths annually. Moreover, biofilms have been estimated to cause $80 \%$ or more of all microbial infections in humans. [4-5]

Biofilms protect their constituent cells in various ways, which makes both clinical and industrial contamination difficult to treat. As self-organized communities, biofilms have evolved to feature differentiated cell phenotypes performing complimentary functions. The associated cooperative behavior of bacterial cells, mediated by cell-cell communications and other factors, enables an increased metabolic diversity and efficiency as well as an enhanced resistance to environmental stress, antimicrobial agents, and the host's defenses. [6-7] Biofilms organize into spatial patterns at the macroscopic and microscopic level. For example, some constituent cells are active in spreading the biofilm while others enter dormant states invulnerable to antimicrobials based on 
metabolic or reproductive pathways. [8-11] The macroscopic physical properties of biofilms are reported to also protect constituent cells, resisting conventional liquid and vapor-phase treatments. [12]

A wide range of bacterial resistant surfaces have been proposed to inhibit biofilm growth a priori, but the typical strategies rely either on a release of biocidal compounds or on inhibiting adhesion. In the first case, traditional techniques involve the design of coatings that release agents such as antibiotics, quaternary ammonium salts, and silver ion into the surrounding aqueous environment. Such agents have been incorporated into a variety of engineering polymers and other materials. [13] The latter approach has focused on the use of surface chemical functional groups that prevent protein adsorption as a means to inhibit bacterial adhesion. One of the most commonly studied such surface modifications is poly(ethylene glycol), or PEG. [14-15]

Both of these strategies, however, are transient. Materials that persistently resist bacteria are difficult to achieve by surface chemistry alone. Even if bacteria are unable to attach directly to a substrate, nonspecific adsorption of proteins and surfactants secreted by bacteria to the surface eventually masks the underlying chemical functionality. [16-18] Over a longer timescale, the reservoir of leaching antimicrobial is normally finite and subject to depletion. Also, the emergence of antibiotic- and silver-resistant pathogenic strains, along with new restrictions on the use of biocide-releasing coatings in the marine environment, have necessitated the development of new strategies [19-21]

In contrast, the effects of topographical features on bacterial adhesion and biofilm formation are poorly understood. Nature provides some clues to preventing microbial colonization of surfaces by this alternative strategy. For example, ship hulls constantly amass layers of algae and crustaceans. Yet materials with topographical features mimicking the skin of sharks have shown increased resistance to marine biofouling at certain length scales. [22] Physical structures may provide a more persistent form of inhibitive interaction between bacteria and surfaces. Indeed, mammalian cells respond to surface topography and mechanics and their behavior can be manipulated using only spatial and mechanical cues. [23-25] Bacteria have been reported to also respond to mechanical cues from the environment. Surface attachment is an integral step in biofilm formation and impacts chemical signaling pathways between and within bacterial cells. [26] Substrate elastic modulus, for example, has been suggested to affect the density of surface colonization. Specifically, on flat surfaces in the Young's modulus range of 1 to $100 \mathrm{MPa}$, a positive correlation is reported between attachment density and surface modulus. [27] Topographical features can influence the arrangement and the resulting behavior of cells on surfaces and may affect biofilm development. [28] However, the roles of specific surface structures in modifying bacterial attachment and subsequent behavior-particularly the role of geometric parameters relative to the mechanical properties of the surface structures - remain unclear.

Here we present new findings on the interactions governing bacterial assembly on nanopost substrates. The attachment and biofilm accumulation behavior were studied by altering the symmetries, dimensions, and pitch (center-to-center distance) of nanopost arrays using a combinatorial approach. Additionally, the attachment of bacteria to nanostructured substrates as a function of the effective stiffness was investigated and compared to the attachment to flat, unpatterned surfaces. Rather than rely on intrinsic material modulus, we exploit a derived effective stiffness, dependent on both the material 
and geometric properties of the surface, to direct bacterial adhesion and to affect biofilm growth.

\section{Experimental}

\subsection{Substrate fabrication}

Nanostructured substrates were fabricated with feature dimensions on the order of the size of bacterial cells. Arrays of high-aspect ratio nanometer-scale polymer posts were generated in UV-curable epoxy (Epoxy Technology UVO-114) and polyurethanes (Norland Optical Adhesives (NOA) 61 and 65) using fast polymer replication technique described previously. [29] Sets of replicas with varying dimensional parameters, including nanopost diameter, pitch, and array symmetry, were molded using different microfabricated Si masters and geometric manipulations of polydimethylsiloxane (PDMS, Dow Corning Sylgard 184) molds.

Several experiments used a combinatorial substrate of posts in an array of square symmetry with a variation of the pitch of posts from one end of the substrate to the other. To make the rectangular symmetry post array with a pitch gradient, a replica was made from a uniaxially stretched PDMS mold with a square array pitch gradient pattern. The mold was stretched and the epoxy was cured on the stretched mold. The array consisted of posts having a diameter of about $300 \mathrm{~nm}$ and a height of about $2 \mu \mathrm{m}$.

Post substrates with orthogonal post pitch and diameter gradients were fabricated using the STEPS method described previously. [30] An epoxy (UVO-114) replica of a nanopost array, containing a pitch gradient from 4.0 down to $0.9 \mu \mathrm{m}$ across the substrate, was sputter coated with $50 \mathrm{~nm}$ of Au to make the surface electrically conductive. The gold-coated sample was then $80 \%$ submerged into an electrochemical cell with $0.1 \mathrm{M}$ pyrrole monomer and $0.1 \mathrm{M}$ sodium dodecylbenzensulfonate as electrolyte. Potentiostatic $(0.55 \mathrm{~V}$ vs. $\mathrm{Ag} / \mathrm{AgCl})$ electrodepositions of polypyrrole were employed with stepwise withdrawal of the sample in the direction perpendicular to the pitch gradient in four equal, discrete steps at $t=15,30,45$, and 60 minutes (Figure 2a). Between time points the sample was stationary with 30 minutes of equilibration and drying time. Since the withdrawal direction was perpendicular to the pitch gradient, an orthogonal gradient of nanostructure diameter corresponding to the PPy growth time was obtained.

Polymer replicas consisting of square post arrays were also fabricated from UVO114 , NOA 61 , and NOA 65 - to vary substrate stiffness - by curing under UV lamps at $130 \mathrm{~mW} / \mathrm{cm}^{2}$ for 20,60 , and 60 minutes respectively. These arrays had dimensions of 2 $\mu \mathrm{m}$ pitch, $250 \mathrm{~nm}$ diameter, and $8 \mu \mathrm{m}$ tall posts with a projected area of $4 \mathrm{~cm}^{2}$. Flat substrates of equal area were fabricated from the same polymers to serve as controls.

\subsection{Measurement of Young's modulus}

The Young's moduli of the NOA 61 and NOA 65 polymers were determined by the fourpoint flexure method previously described [29], except that the flexure samples were fabricated, in triplicates, with the following dimensions: length $=40 \mathrm{~mm}$, width $=5 \mathrm{~mm}$, thickness $=1.5 \mathrm{~mm}$, and the mechanical measurements were performed on an Instron 5566 universal test system. 


\subsection{Bacterial preparation and growth}

Pseudomonas aeruginosa (strain PA14) was grown in Luria broth (LB) media (EMD LB Broth Miller) overnight at $37^{\circ} \mathrm{C}$ in loosely capped tubes on an orbital shaker to the stationary phase. This LB preculture was then seeded at $1 \%$ concentration in tryptone broth (TB) growth media (BD Bacto Tryptone) on polymer substrates attached with carbon tape to the bottoms of six-well plates. Samples were immersed in $4 \mathrm{~mL}$ of inoculated medium and grown on the bench at room temperature for various time points.

\subsection{Imaging and analysis}

For fluorescence imaging of attached bacterial cells following a growth period, the growth culture was aspirated from each well, the submerged polymer samples were gently rinsed in the well with phosphate buffered saline (PBS) (1x) (Lonza Biowhittaker), and the adherent bacteria were fixed by $5 \%$ glutaraldehyde solution for at least 1 hour. Following another PBS rinse, 0.01\% Triton X100 in PBS (PBST) was used to permeabilize the bacteria membranes. Next the cells were stained with $0.5 \mu \mathrm{M}$ SYTOX green nucleic acid stain (Invitrogen) in PBST for 30 minutes, after which they were rinsed in PBS for fluorescence imaging.

To analyze the ordering present in different fluorescence micrographs, Fast Fourier Transforms (FFT) were performed and contrast-optimized using ImageJ.

\subsection{Bacterial quantification by sonication and colony forming units $C F U$}

The attached bacteria or biomass on various polymer samples was quantified using a multi-step process of cell removal, serial dilution, and plating for viable cell counts. First, each polymer sample was individually placed into a $50 \mathrm{~mL}$ conical tube containing $15 \mathrm{~mL}$ of $3 \mathrm{mM}$ D-tyrosine in PBS at $\mathrm{pH} 7.5$ and incubated at room temperature for 30 minutes to promote biofilm disassembly. Based on results from peakCFU optimization experiments, the samples were bath-sonicated for 10 minutes. $200 \mu \mathrm{L}$ of each dilute bacterial solution was pipetted into a 96-well plate and serially 10 -fold diluted. The serial dilutions of each sample were pipetted in parallel onto an LB agar plate, which was briefly tilted to spread the drops into parallel lines and was then incubated for 36-48 hours at room temperature. CFU were visually counted and compared at corresponding dilution factors.

\section{Results and Discussion}

\subsection{Topographical effects on the alignment of bacteria}

The replication method used to fabricate the polymer high-aspect-ratio (HAR) nanopost substrates can be modified to create post arrays with a range of dimensions and symmetries from the same elastomeric molds. [29] Applying precise sets of deformations to the elastomeric molds while the replica material is cured in them allows nanopost cross-sections to be proportionally elongated, nanoposts to be tilted away from 
the vertical axis, and the array to be transformed from square to rectangular or rhombic. Additionally, substrate curvature or twist can be introduced. These nontrivial substrate modifications are possible either through simultaneous mold deformations or through iterative mold deformation and replication steps.

\subsubsection{Directed cell alignment by topographical cues}

In particular, the four-fold symmetry of the square post array used in pitch gradient samples can be broken by uniaxial extension of the mold during the substrate curing step. In this manner, the square post array is transformed into a rectangular array, where the post pitch is larger in one lattice direction than in the orthogonal lattice direction due to Poisson compression (Figure 1a). Previous work demonstrated the spontaneous assembly of bacteria on periodic post arrays and suggested a model for bacterial patterning by surface contact area maximization. [28] With this principle in mind, we designed substrates to align bacterial adhesion on surfaces using only topographical cues.

Using uniaxial extension of a surface with square symmetry and a spatial post pitch gradient across the substrate during the curing step, we generated a rectangular post array with spatially varying pitch along both lattice parameters. P. aeruginosa was cultured on these anisotropic HAR nanopost arrays, and their self-assembled patterns were observed by fluorescent microscopy (Figure 1b). Consistent with previous results [28], the bacteria attach in different configurations depending on the pitch of the posts. At the small-pitch extreme $(0.9 \mu \mathrm{m})$ the bacteria align themselves with the posts, oriented normal to the substrate, forming a rectangular array on the surface. As the post pitch increases the bacteria align themselves in the orthogonal directions of symmetry of the array parallel to the substrate.

The organizational characteristics of the bacterial patterns are more clearly seen in the Fourier transforms (FFT) of the fluorescence intensity images (Figure 1c). The small points correspond to positional ordering peaks and the anisotropy of the diffuse central spot corresponds to the orientational ordering of the rod-like cells. The positional ordering peaks are spaced at larger distances along the [01] vertical direction (corresponding to smaller post spacing in real-space) than in the [10] horizontal directions, indicating cellular registration with the rectangular post symmetry. Within the closely spaced posts, where the cells align normal to the substrate, the positional ordering peaks reflect the rectangular symmetry of the bacterial pattern, but the central spot is isotropic. This lack of orientational order in the FFT image is a result of the fact that the cells are oriented parallel to the angle of viewing, and when viewed along their long axis, the rod-like cells lack orientational anisotropy. Moving across the substrate, as the posts get farther apart, the positional ordering peaks get correspondingly closer together, and the central spot becomes anisotropic as the cells lie parallel to the substrate and normal to the viewing angle. Further across the pitch gradient, there is a spacing at which bacteria can bridge neighboring posts along the contracted [01] direction and not along the stretched [10] direction of the array. Due to their contact area maximization behavior, the bacteria preferentially lie in the contracted direction, as can be seen by the vertical lines in the fluorescence image (Figure 1b) and in the anisotropic stretching of the central spot in the same direction (Figure 1c). This uniaxial orientational ordering confirms that the 
bacteria adopt specific and robust configurations when adhering to the substrate and demonstrates the influence of surface topography on bacterial assembly.

\subsubsection{Topographical features direct bacterial order and disorder}

By precisely controlling the topography of the post array, we further demonstrate the ability to drive long-range bacterial assembly from a disordered to an ordered state and vice versa. Starting with a HAR nanopost array that had been fabricated with a pitch gradient, we have fabricated a unique two-dimensional gradient substrate by adapting the Structural Transformation by Electrodepostion of Polymers (STEPS) technique [30], as shown in Figure 2a and described in Experimental. A gradient of nanopost diameters was thus superimposed in the orthogonal direction, creating a 2-D combinatorial array as shown in Figure 2b. Epoxy replicas of the combinatorial array were then fabricated using a double-casting soft lithography method. [29] P. aeruginosa cultures were grown to 18 hours on the epoxy replicas, and the resulting attachment was studied along the pitch and diameter gradients of the substrate by fluorescence microscopy. Pronounced effects of both post pitch and diameter on the mode of bacterial attachment were observed.

For large-pitch regions of the array, increasing nanopost diameter drives the bacterial patterning to order, first in the [01] and [10] in-plane directions on the substrate, and with further increased diameter, the bacteria begins to orient out-of-plane. This result recapitulates the effect of decreasing post pitch, showing that the wall-to-wall gap (pitch minus diameter), rather than the pitch or diameter per se, is the critical ordering parameter. The in-plane configuration is maximized with a gap of approximately $\mathrm{a}_{10}=$ $0.90 \mu \mathrm{m}$. Correspondingly, the bacteria attaching on a region of the substrate with smaller pitch were observed to first pattern in the [01], [10] arrangement, then assemble into the out-of-plane configuration with increasing diameter; diameter-pitch combinations producing a [11] gap dimension of about $1.05 \mu \mathrm{m}$ maximize this configuration. Finally, the bacteria attachment becomes random and disordered as the spacing between posts further diminishes. At this point, the interstitial space is insufficient for the cells to insert between nearest neighbor posts. Conversely, no orientational ordering results from combinations of pitch and native diameter in which the spacing is larger than the length of the bacterial cells, as expected from the contact area maximization behavior model. [28] The fluorescence microscopy images and corresponding FFTs in Figure 3a-c demonstrate these finely controlled transitions between order to disorder, increasing order, and disorder to order. Moreover, the length scale of the gap for optimal ordering closely correlates to the length scale of the bacterium, which is about $1 \mu \mathrm{m}$. Therefore, by designing a HAR nanostructured substrate with the appropriate length scale interstices, bacteria can be induced to order in arbitrary patterns.

\subsection{Mechanical effects of nanostructured surfaces on biofilm growth}

In addition to controlling bacterial attachment to surfaces through the use of nanostructures and nanotopography, the mechanical properties of a surface have recently been reported to play a role [27], similar to mechanoselective adhesion observed in mammalian cells. For bacteria, this phenomenon has been shown in conjunction with flat unpatterned surfaces in the Young's modulus range of $\sim 1$ to $\sim 100 \mathrm{MPa}$, specifically that 
there is a strong positive correlation between attachment density and surface stiffness. The stiffness in this case is modulated by the $\mathrm{pH}$ during fabrication of the polyelectrolyte multilayers. However, a bacteria-inhibitive material in the $1 \mathrm{MPa}$ or lower stiffness regime may not be practical for some technological or device applications due to low mechanical rigidity. A monolithic material rather than a surface coating would also not be susceptible to potential delamination. In this study, we use HAR surface nanoarrays comprised of the bulk material to emulate a thin, ultra-compliant surface coating. Suspended bacteria present near the nanoarray surface interact with highly flexible cantilevers rather than with a flat surface. The most flexible nanoposts in our study deflect $\sim 5 \mu \mathrm{m}$ per $100 \mathrm{pN}$ of force applied to the tip perpendicular to the long axis of the post, based on Eulerian beam mechanics: $\delta=\frac{F l^{3}}{3 E I}$, where $\delta$ is the tip deflection, $F$ applied force, $l$ post height, $E$ elastic modulus, and $I$ moment of inertia. By comparison, for a bacterium to indent a flat surface $\sim 5 \mu \mathrm{m}$ per $100 \mathrm{pN}$, the elastic modulus would need to be $\sim 5 \mathrm{~Pa}$. For this, we assume the $200 \mathrm{~nm}$ radius cells to apply force with their hemispherical poles and we use the Hertzian elastic contact model for indentation: $\delta=\left(\frac{4 E}{3 F\left(1-v^{2}\right)} R^{0.5}\right)^{2 / 3}$, with $\delta$ the indentation depth, $v$ Poisson's ratio, $E$ elastic modulus, $F$ applied force, and $R$ indenter radius. By this analysis, the soft, hair-like surface naïvely attains effective stiffnesses six orders of magnitude lower than the constituent polymers, which are themselves in the range of 20 to $2000 \mathrm{MPa}$.

\subsubsection{Normalizing biofilm growth on polymer surfaces}

Using the HAR nanoarray platform and a CFU biofilm quantification assay (see Methods), we investigated the attachment density of bacteria on surfaces as a function of the nanoarray effective stiffness. The effective stiffness was controlled by selection of the polymer so as to keep the nanoarray geometry constant. Six candidate polymer systems were first screened for leaching toxicity to $P$. aeruginosa. Flat substrates made of each polymer were sputter coated with $20 \mathrm{~nm}$ Au to mask their native surface chemistry, and biofilms cultured on these substrates were quantified (Figure S1). The polyurethanes NOA65, $20 \mathrm{MPa}$; NOA61, $500 \mathrm{MPa}$; and the epoxy UVO114, $2000 \mathrm{MPa}$ were selected for their low leaching toxicity (as measured by high viable cell counts), photocurability, and broad range of modulus values. The relative cytophilicity of each unsputtered polymer was similarly determined, and the subsequent raw data in the biofilm quantifications from nanoarrays and flat substrates across the three materials were normalized.

\subsubsection{Biofilm growth response to the effective stiffness of topographical features}

As seen in Figure 4a-b, the numbers of (CFU) measured from 27-hour biofilms cultured on $2 \mathrm{GPa}$ and $500 \mathrm{MPa}$ nanoarrays were larger than on the corresponding flat surfaces. This trend may be expected based on the nanoarrays' larger surface area versus flat substrates. However, a biofilm growth crossover regime occurs for the $20 \mathrm{MPa}$ NOA65 polymer nanoarray. On this ultra-compliant structured surface, despite the 
surface area increase, resultant biofilm actually decreases with respect to the addition of HAR posts. Since the material cytophilicities are already accounted for, we can conclude that the extremely low effective stiffness experienced by cells interacting with the soft, hair-like surface inhibits biofilm accumulation. (By comparison, P. aeruginosa forms indistinguishably robust biofilm after 24 hours on a polymer surface regardless of the surface chemical functionality, as seen in Figure S2, suggesting its indifference to surface chemistry alone.) Based on the flexible cantilever model of nanoarray effective stiffness discussed above, the minimum threshold for this mechanoselective behavior is on the order of $5 \mu \mathrm{m}$ surface deformation per $100 \mathrm{pN}$ force-equivalent for a bacterium attaching to a flat surface with an elastic modulus of $\sim 5 \mathrm{~Pa}$.

In contrast, the growth crossover regime is not observed once a $20 \mathrm{~nm}$ gold layer is applied to the same three polymer nanoarrays, as shown in Figure 4b. The estimated effect of the gold layer, whose modulus is $79 \mathrm{GPa}$, is to stiffen by about $20 \mathrm{x}$ the polymermetal core-shell nanoposts. With this increase in the HAR nanoarray effective stiffness, the growth crossover in the $20 \mathrm{MPa}$ polymer nanoarray is not observed, suggesting the upper bound of a biofilm growth inhibition range. These effective stiffness values are themselves lower estimates, as bacteria are in contact with not only the distal ends of the nanoposts, but also the geometrically stiffer basal ends, and indeed, with the basal plane. The "average" effective stiffness of these surfaces, at which the mechanoselective adhesion phenomenon occurs, can be assumed to be higher.

Regardless, the passively dynamic nature of such surfaces may present an unstable and unfavorable attachment target for bacteria. Their deformation under imposed bacterial forces can cause them to read as a fluid-like solid, whereas biofilms seek secure and immobile surfaces to colonize. Therefore, the principle of effective stiffness-mediated inhibition of bacterial attachment may constitute promising surface treatments in diverse applications for preventing biofilm accumulation.

\section{Conclusions}

By applying a novel combinatorial platform, we have elucidated key nanometer-scale geometric and mechanical parameters of surfaces that drive long-range bacterial adhesion patterns and biofilm growth behavior. The interstitial spacing between surface features is experimentally confirmed to be the critical parameter controlling assembly, and it can be used to induce specific ordering phases using a range of feature sizes on the order of the bacterial cell. Furthermore, for the first time, the mechanoselective bacterial attachment of bacteria on compliant high-aspect-ratio nanostructures has been described. This extends the recent finding of bacterial mechanosensitivity to a much lower effective stiffness regime of $\sim 50 \mu \mathrm{m} / \mathrm{nN}$, equivalent to flat films with modulus as low as $5 \mathrm{~Pa}$. Moreover, it suggests a completely new strategy to exploit mechanoselective adhesion. When the effective compliance of the hair-like nanoarray is increased beyond a threshold, biofilm growth is inhibited as compared to a flat control surface. As this is strictly a mechanical-structural property and does not rely on surface chemical functionalization, it is not susceptible to masking and may be persistent. As a potential new strategy, HAR nanoarrays mimicking an extremely compliant flat surface offer promise for diverse applications for controlling and inhibiting biofilm accumulation. 


\section{Acknowledgments}

We thank Dr. Yunrong (Win) Chai, Dr. Sigolene Lecuyer, and Anna Wang for biological technique and the BASF Advanced Research Initiative for funding.

\section{References}

1. Shapiro, J., Thinking about bacterial populations as multicellular organisms. Annual Reviews in Microbiology, 1998. 52(1): p. 81-104.

2. Costerton, J.W. and P.S. Stewart, Battling biofilms - The war is against bacterial colonies that cause some of the most tenacious infections known. The weapon is knowledge of the enemy's communication system. Scientific American, 2001. 285(1): p. 74-81.

3. Dalley, A.A.C.a.R., Barnacle fouling and its prevention, in Barnacle Biology, A.J. Southward, Editor. 1987, A. A. Balkema: Rotterdam, NL. p. 419-433.

4. Davies, D., Understanding biofilm resistance to antibacterial agents. Nature Reviews Drug Discovery, 2003. 2(2): p. 114-122.

5. Klevens, R., et al., Estimating health care-associated infections and deaths in US hospitals, 2002. Public Health Reports, 2007. 122(2): p. 160.

6. Marsh, P., Dental plaque: biological significance of a biofilm and community life style. Journal of clinical periodontology, 2005. 32: p. 7-15.

7. Blango, M. and M. Mulvey, Bacterial landlines: contact-dependent signaling in bacterial populations. Current Opinion in Microbiology, 2009. 12(2): p. 177-181.

8. Ben-Jacob, E., I. Cohen, and D.L. Gutnick, Cooperative organization of bacterial colonies: from genotype to morphotype. Annual Reviews in Microbiology, 1998. 52(1): p. 779-806.

9. Klausen, M., et al., Involvement of bacterial migration in the development of complex multicellular structures in Pseudomonas aeruginosa biofilms. Molecular microbiology, 2003. 50(1): p. 61-68.

10. Stewart, P.S. and M.J. Franklin, Physiological heterogeneity in biofilms. Nature Reviews Microbiology, 2008. 6(3): p. 199-210.

11. Vlamakis, H., et al., Control of cell fate by the formation of an architecturally complex bacterial community. Genes \& development, 2008. 22(7): p. 945.

12. Epstein, A., et al., Bacterial biofilm shows persistent resistance to liquid wetting and gas penetration. Proceedings of the National Academy of Sciences, 2010.

13. Banerjee, I., R. Pangule, and R. Kane, Antifouling Coatings: Recent Developments in the Design of Surfaces That Prevent Fouling by Proteins, Bacteria, and Marine Organisms. Advanced Materials, 2010.

14. Park, K.D., et al., Bacterial adhesion on PEG modified polyurethane surfaces. Biomaterials, 1998. 19(7-9): p. 851-859.

15. Prime, K.L. and G.M. Whitesides, Self-assembled organic monolayers: model systems for studying adsorption of proteins at surfaces. Science (New York, NY), 1991. 252(5010): p. 1164.

16. Bos, R., et al., Retention of bacteria on a substratum surface with micro patterned hydrophobicity. Fems Microbiology Letters, 2000. 189(2): p. 311-315.

17. Gristina, A., Biomaterial-centered infection: microbial adhesion versus tissue integration. Science, 1987. 237(4822): p. 1588. 
18. Neu, T., Significance of bacterial surface-active compounds in interaction of bacteria with interfaces. Microbiology and Molecular Biology Reviews, 1996. 60(1): p. 151.

19. Hall-Stoodley, L., J.W. Costerton, and P. Stoodley, Bacterial biofilms: from the natural environment to infectious diseases. Nature Reviews Microbiology, 2004. 2(2): p. 95-108.

20. Trevors, J., Silver resistance and accumulation in bacteria. Enzyme and Microbial Technology, 1987. 9(6): p. 331-333.

21. Costerton, J., P. Stewart, and E. Greenberg, Bacterial biofilms: a common cause of persistent infections. Science, 1999. 284(5418): p. 1318.

22. Schumacher, J., et al., Engineered antifouling microtopographies-effect of feature size, geometry, and roughness on settlement of zoospores of the green alga Ulva. Biofouling, 2007. 23(1): p. 55-62.

23. Discher, D., P. Janmey, and Y. Wang, Tissue cells feel and respond to the stiffness of their substrate. Science, 2005.310(5751): p. 1139.

24. Huebsch, N., et al., Harnessing traction-mediated manipulation of the cell/matrix interface to control stem-cell fate. Nature Materials, 2010.

25. Stevens, M.M. and J.H. George, Exploring and engineering the cell surface interface. Science, 2005. 310(5751): p. 1135-1138.

26. Davey, M. and G. O'toole, Microbial biofilms: from ecology to molecular genetics. Microbiology and Molecular Biology Reviews, 2000. 64(4): p. 847.

27. Lichter, J., et al., Substrata mechanical stiffness can regulate adhesion of viable bacteria. Biomacromolecules, 2008. 9(6): p. 1571-1578.

28. Hochbaum, A. and J. Aizenberg, Bacteria Pattern Spontaneously on Periodic Nanostructure Arrays. Nano Letters, 2010.

29. Pokroy, B., et al., Fabrication of bioinspired actuated nanostructures with arbitrary geometry and stiffness. Advanced Materials, 2009. 21(4): p. 463-469.

30. Kim, P., et al., Structural Transformation by Electrodeposition on Patterned Substrates (STEPS): A New Versatile Nanofabrication Method. Nano Letters, 2011.

\section{Captions}

Fig. 1 (a) Schematic of uniaxial elastomeric mold stretching and the resultant nanopost array transformation from square to rectangular symmetry. (b) Fluorescence images of $P$. aeruginosa bacteria following 18 hour growth on nanopost substrates that range from square symmetry, at left, to increasingly elongated rectangular symmetry, at right. The vertical [01] lines of bacteria, corresponding to the smallest interstitial space, is consistent with surface contact area maximization as a driver for spontaneous attachment. (c) From left to right, fast Fourier transforms of increasingly anisotropic high-aspect-ratio nanopost arrays visualize the induced directional patterning of bacteria. The elongation of the central spot indicates that bacteria preferentially lie along the close-packed [01] direction.

Fig. 2 (a) Schematic of the orthogonal double gradient substrate fabrication, adapting the recently described STEPS method [30]. A high-aspect-ratio nanopost array with a pitch 
gradient along one axis was withdrawn in discrete steps in the perpendicular direction from the electrodeposition bath, resulting in discrete increases in nanopost diameter. This substrate was then replicated multiple times using a fast double-molding method [29].

(b) The final combinatorial substrate includes both a pitch $(p)$ gradient from $0.8 \mu \mathrm{m}$ to 4.0 $\mu \mathrm{m}$ and an orthogonal nanopost diameter (d) gradient from $300 \mathrm{~nm}$ to $\sim 1 \mathrm{um}$. The interstitial spaces $\mathrm{a}_{[10]}$ and $\mathrm{a}_{[01]}$ are respectively the difference of $p-d$ in the [10] and [01] directions.

Fig. 3 Fluorescence images and corresponding Fourier transforms of spontaneously patterned P. aeruginosa bacteria on the combinatorial nanoarray substrate. The image sequences show attachment along regions of equal pitch and increasing diameter, from top to bottom. The interstitial spacing, not center-to-center pitch, of the HAR nanoarray is the functional surface parameter for spontaneous bacterial patterning on the surface. By precisely tuning nanoarray geometry, the effect can be controlled. (a) An order to disorder transition is induced as the $\mathrm{a}_{11}$ interstitial space is decreased to the point that bacteria can no longer insert between nearest neighbor nanoposts. (b) Initially in-plane bacteria transition to out-of-plane order as the interstitial space becomes optimized for out-of-plane insertion. (c) Initially disordered bacteria transition to a high degree of order.

Fig. 4 (a) Colony forming unit (CFU) measurements of $P$. aeruginosa biofilm growth on both unpatterned and high-aspect-ratio (HAR) nanopost substrates fabricated from polymers of varying Young's modulus. The UVO114 (2 GPa) and NOA61 (500 MPa) nanopost substrates show increased growth versus the unpatterned control substrates. However, a biofilm growth crossover regime with respect to the addition of HAR nanoposts occurs in the softest polymer, NOA65. Despite a surface area increase, resultant biofilm decreases.

(b) The crossover regime is not observed when a $20 \mathrm{~nm}$ stiffening gold coating is applied to the nanoposts, indicating the upper bound of an effective stiffness range for biofilm growth inhibition.

Fig. S1 Leaching and toxicity screening was performed using $20 \mathrm{~nm}$ Au-coated samples of a range of candidate polymers. UVO-114, NOA61, and NOA65 were selected for their low leaching/toxicity, based on high CFU counts, as well as for their photocurability and their range of modulus values.

Fig. S2 Fluorescence images of $P$. aeruginosa bacterial growth on epoxy (UVO-114) flat substrates functionalized with either hydrophobic or hydrophilic surface chemistries. Surface chemistry treatments show only transient effects on biofilm growth. They may influence initial attachment, e.g., at 6 hours, but become irrelevant by longer time scales of interest. 
a.

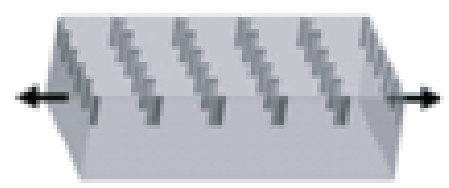

[10]

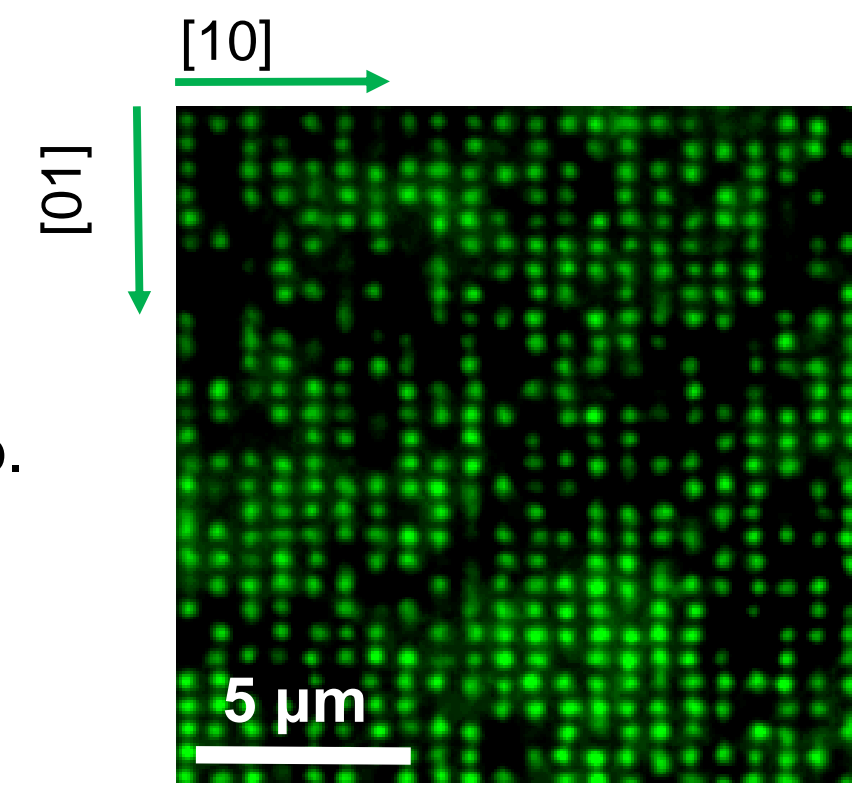

- 0

- $0 \square$ 0 $\square$ 0

- $\circ$

○ 0
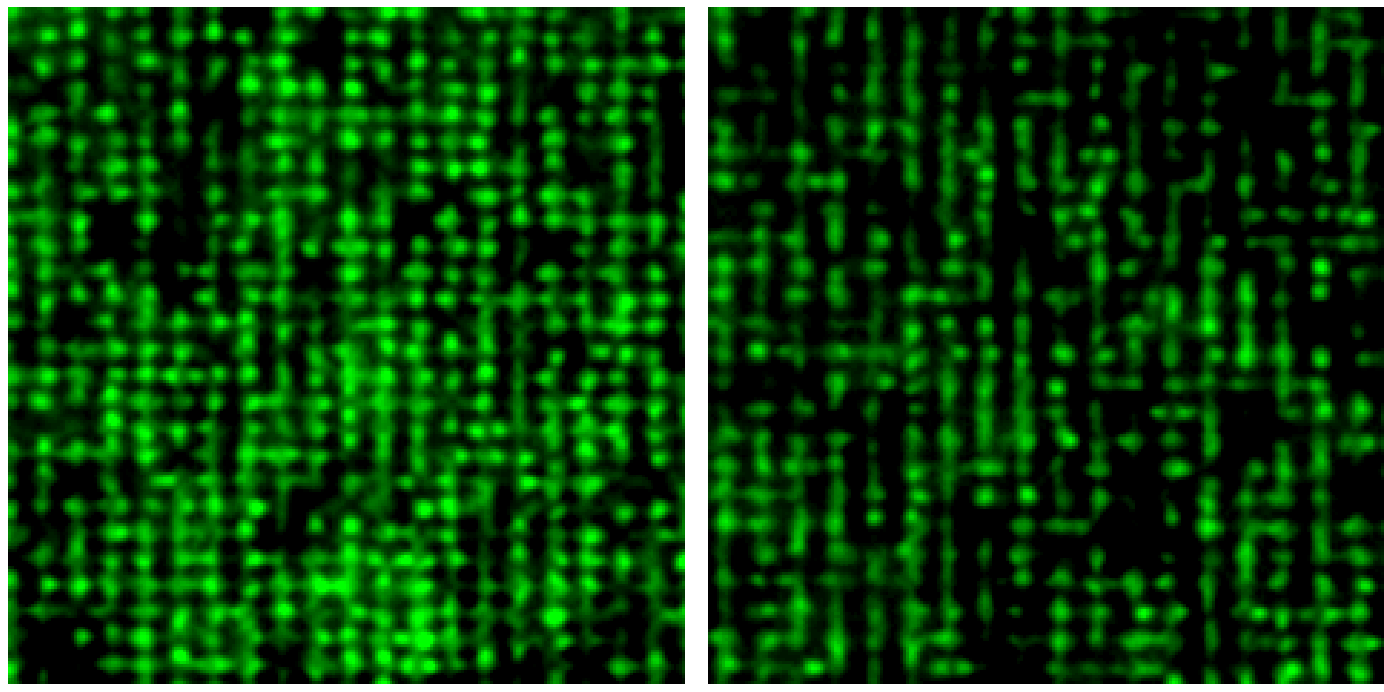

C.
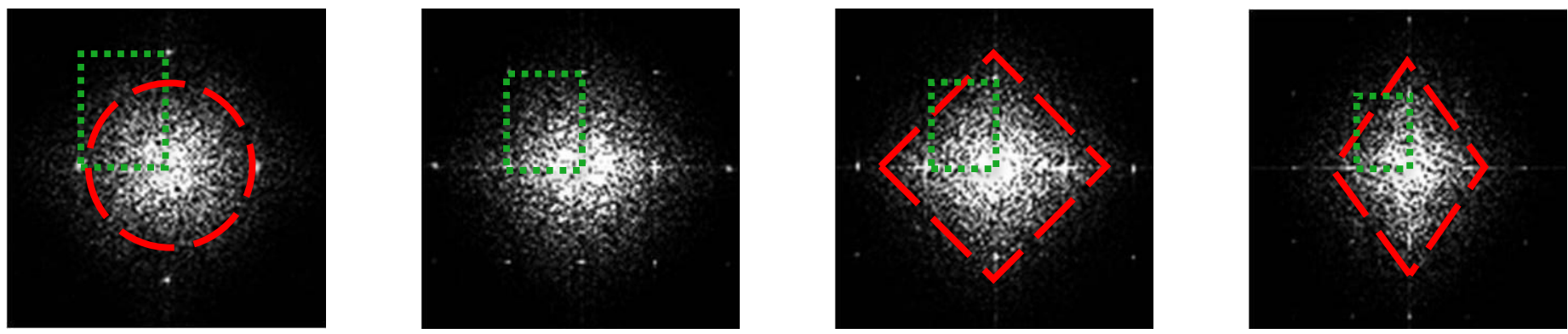
a.

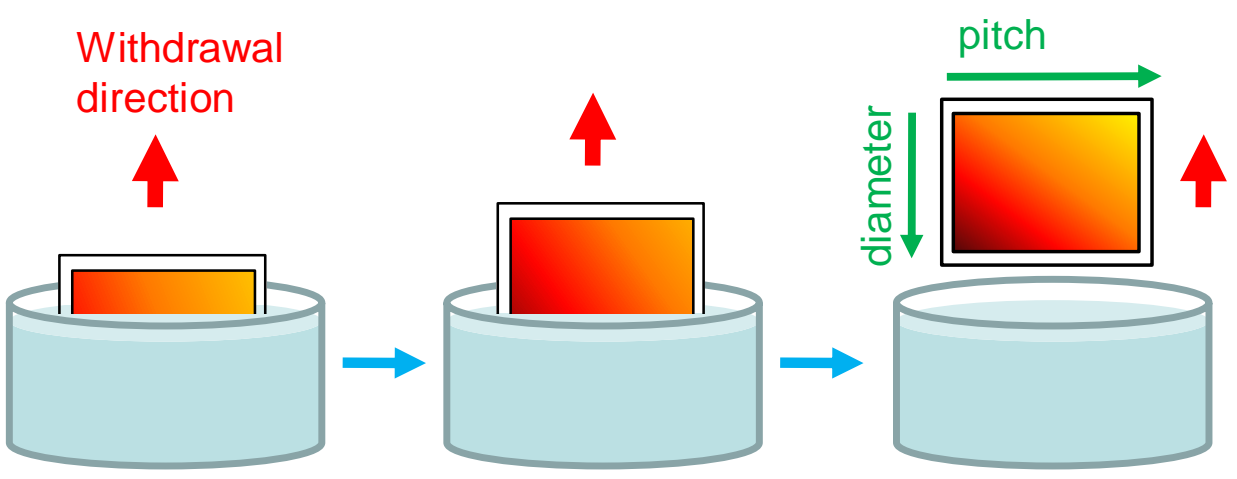

b.

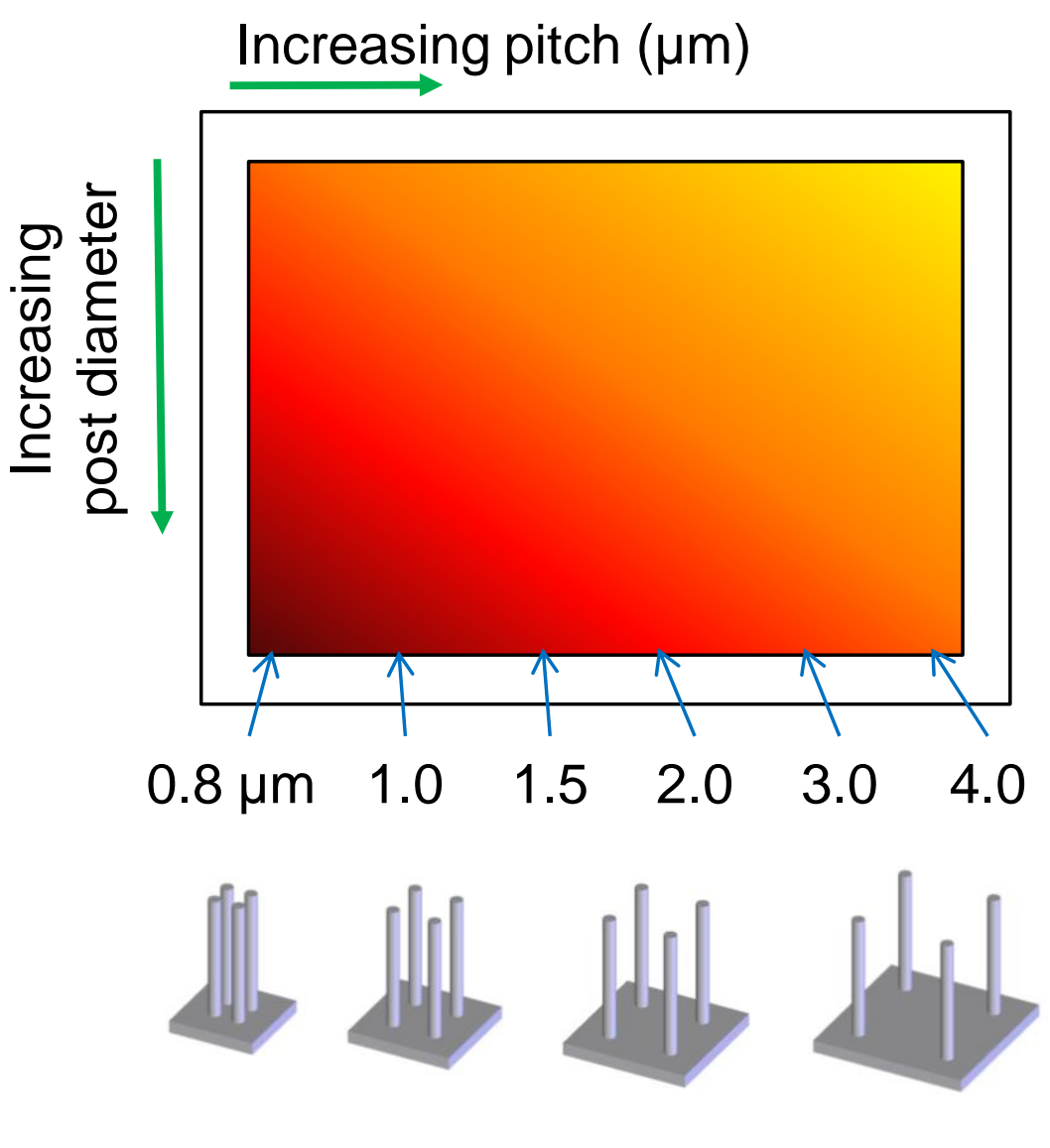


a.

\section{Order to disorder transition}

$P=1.33$ um

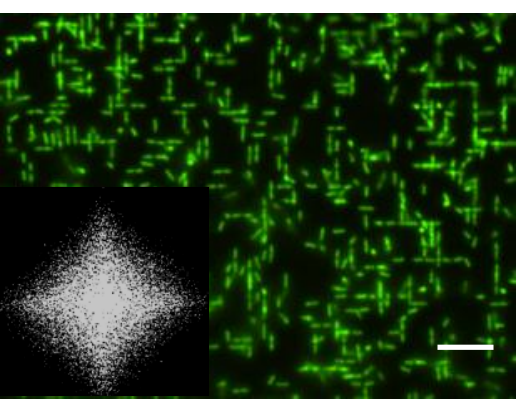

$a_{10}=0.90 \mu \mathrm{m}$

$a_{11}=1.45 \mu \mathrm{m}$

$a_{10}=0.48 \mu \mathrm{m}$

$a_{11}=1.03 \mu \mathrm{m}$

$a_{10}=0.10 \mu \mathrm{m}$

$a_{11}=0.65 \mu \mathrm{m}$
Increasing order transition

$\mathrm{P}=1.66 \mathrm{um}$

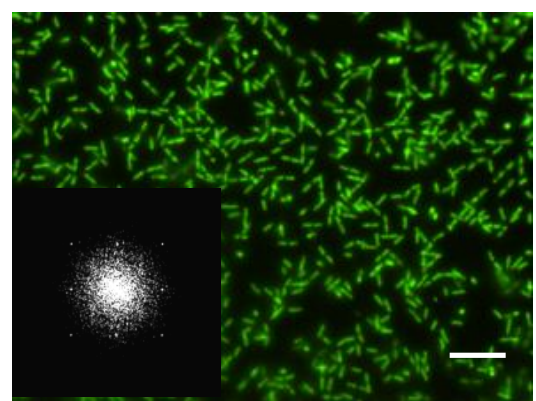

$a_{10}=1.19 \mu \mathrm{m}$

$a_{11}=1.88 \mu \mathrm{m}$

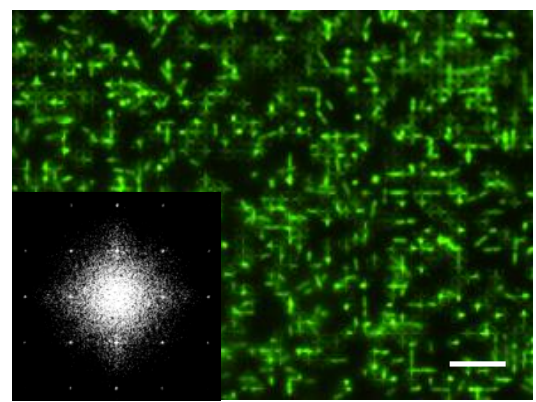

$a_{10}=0.63 \mu \mathrm{m}$

$a_{11}=1.32 \mu \mathrm{m}$

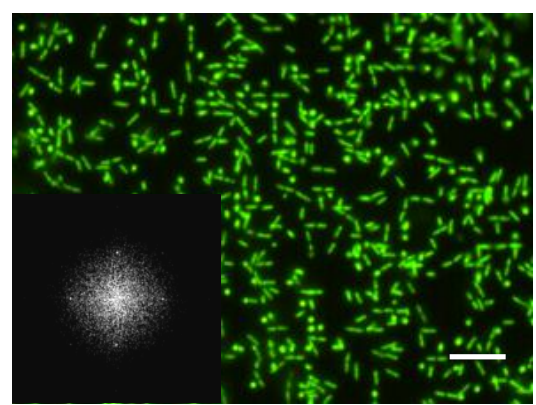

$a_{10}=0.26 \mu \mathrm{m}$

$a_{11}=0.95 \mu \mathrm{m}$
C.

Disorder to order transition

$\mathrm{P}=2.0 \mathrm{um}$

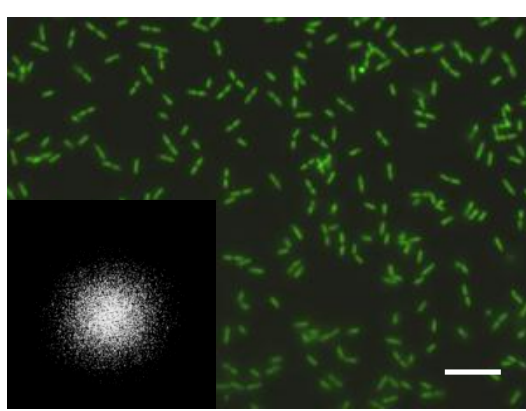

$\mathrm{a}_{10}=1.52 \mu \mathrm{m}$ $\mathrm{a}_{11}=2.35 \mu \mathrm{m}$

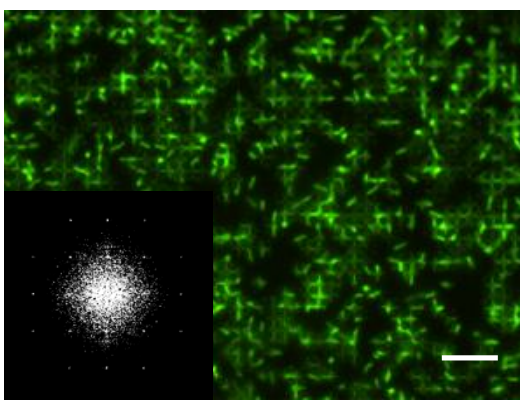

$\mathrm{a}_{10}=0.97 \mu \mathrm{m}$

$a_{11}=1.80 \mu \mathrm{m}$

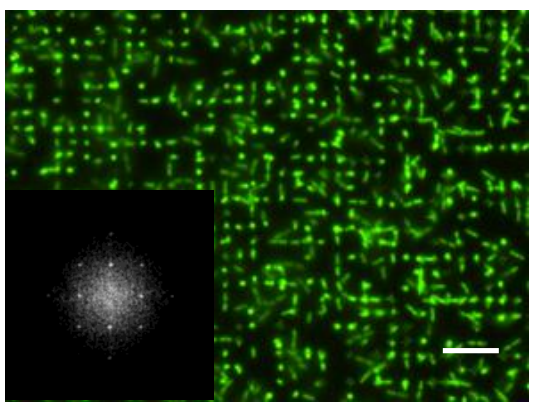

$a_{10}=0.22 \mathrm{um}$

$a_{11}=1.05 \mu \mathrm{m}$

$a_{10}=$ wall-to-wall [10] spacing

$a_{11}=$ wall-to-wall [11] spacing 
a.

CFU on uncoated samples

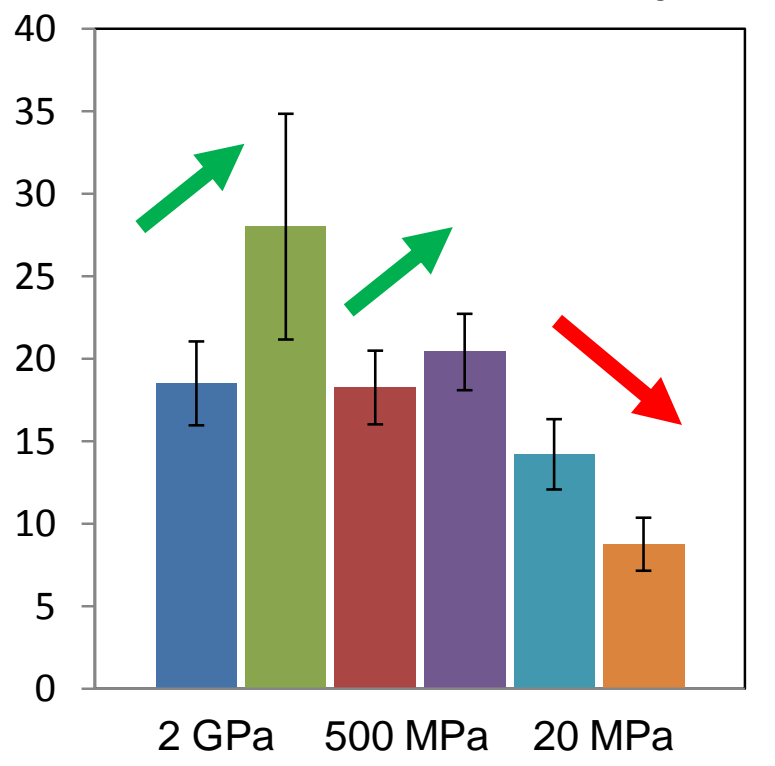

b.

CFU on metal-coated samples

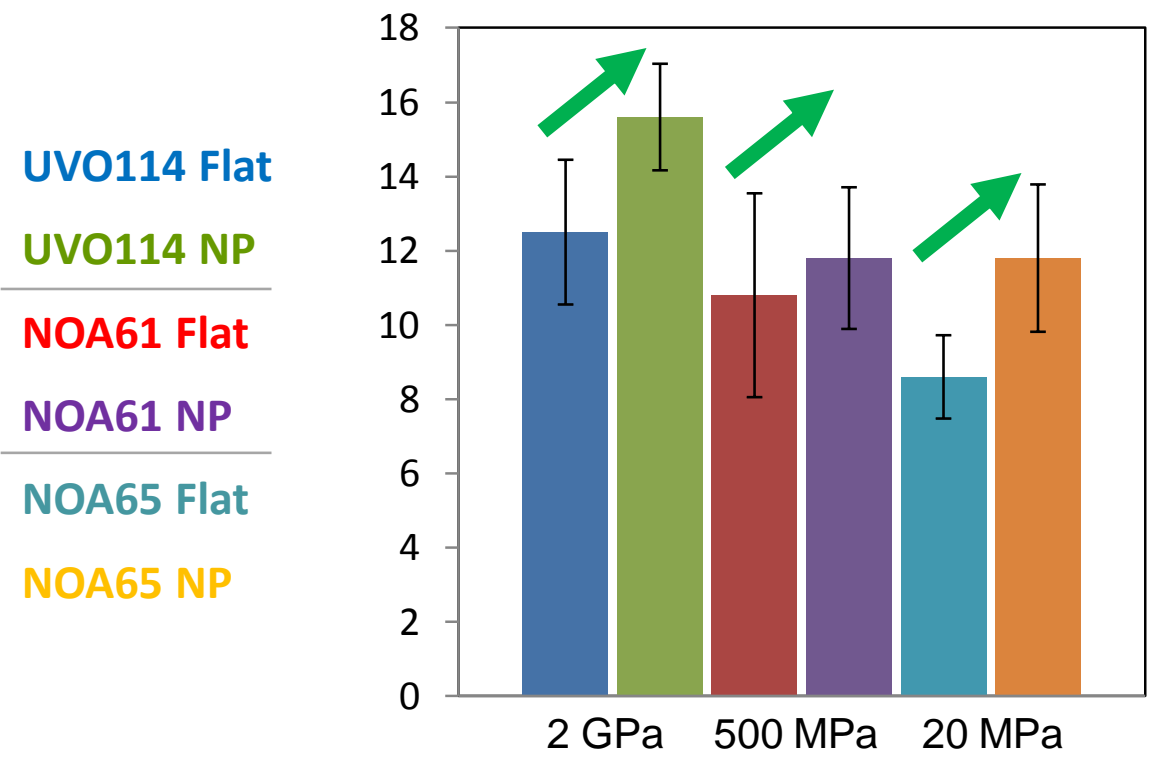




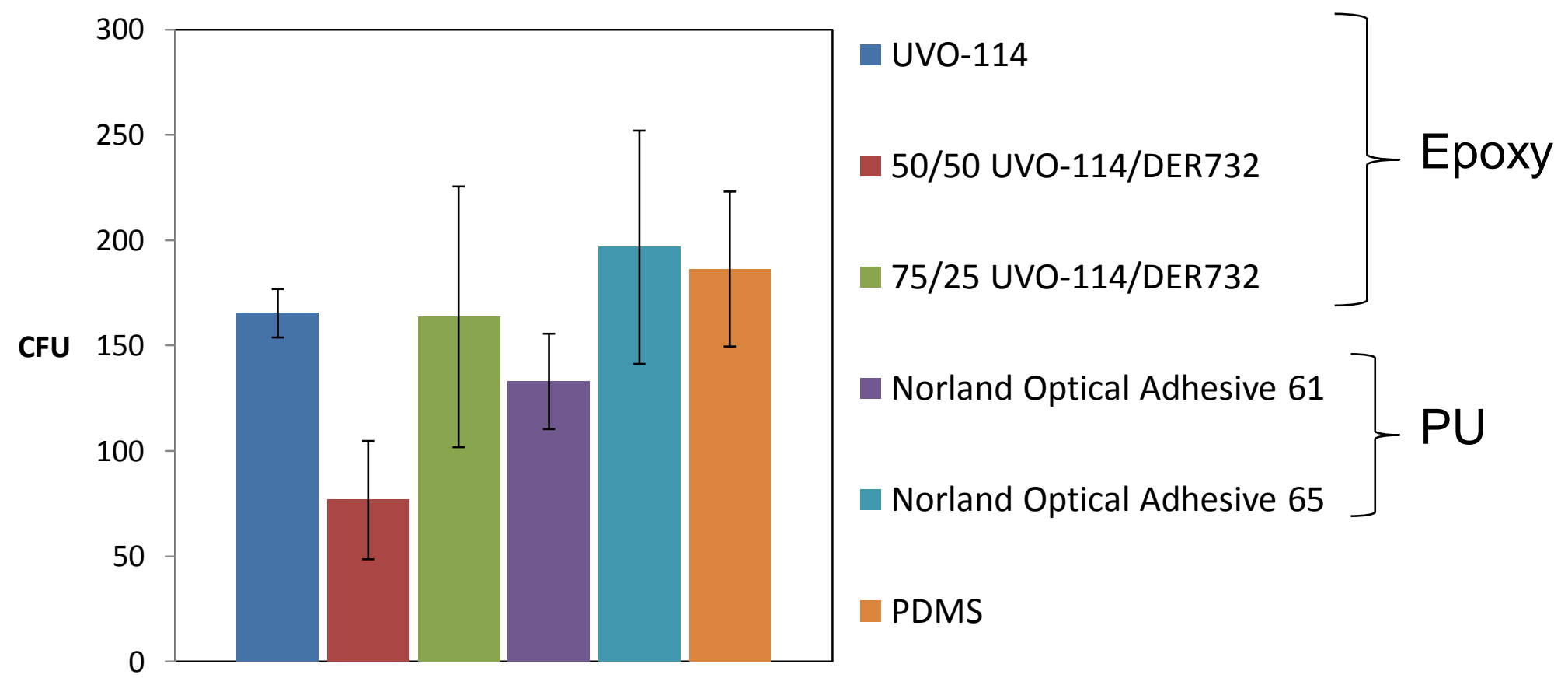




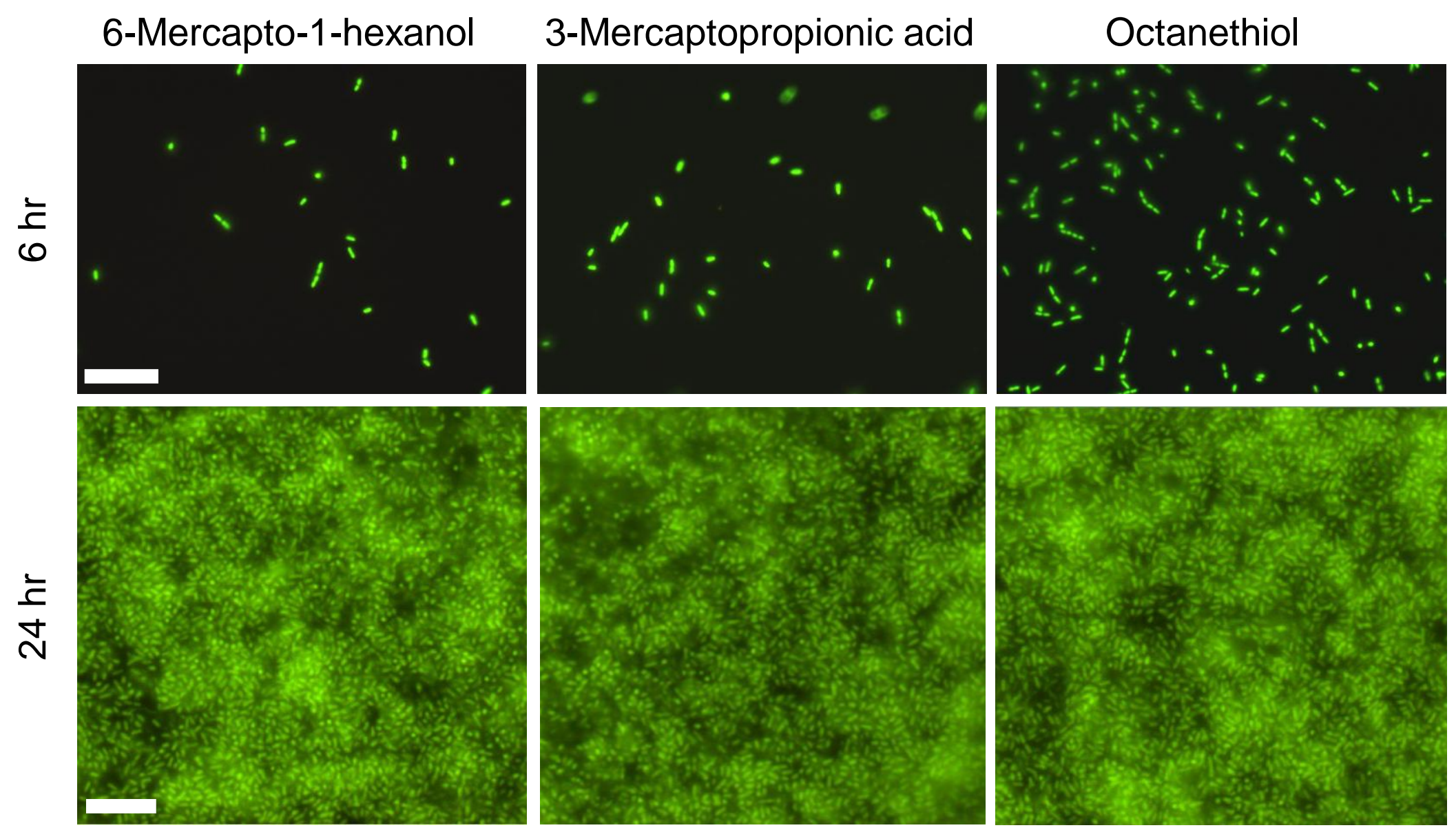

